\title{
ABSTRACT FROM CURRENT LITERATURE
}

\section{Environmental toxic metal contaminants and risk of Cardiovascular disease: systematic review and meta-analysis}

Chowdhury R, Ramond A, O'Keefee LM, et al. BMJ 2018;362: k3310, doi: 10.1136/bmj.k 3310

Objective: To conduct a systematic review and meta-analysis of epidemiological studies investigating the association of arsenic, lead, cadmium, mercury, and copper with cardiovascular disease.

Design: Systematic review and meta analysis.

Data Sources: PubMed, Embase, and Web of Science searched up to December 2017.

Review methods: Studies reporting risk estimates for total cardiovascular disease, coronary heart disease, and stroke for levels of arsenic, lead, cadmium, mercury, or copper were included. Two investigations independently extracted information on study characteristics and outcomes in accordance with PRISMA and MOOSE guidelines. Relative risks were standardised to a common scale and pooled across studies for each marker using random effects meta-analyses.

Results: The review identified 37 unique studies comprising 348259 non overlapping participants, with 13033 coronary heart disease, 4205 stroke, and 15274 cardiovascular disease outcomes in aggregate. Comparing top versus bottom thirds of baseline levels, pooled relative risks for arsenic and lead were 1.30 (95\% confidence interval 1.04 to 1.63$)$ and 1.43 (1.16 to 1.76$)$ for cardiovascular disease, 1.23 (1.04 to 1.45 ) and 1.85 (1.27 to 2.69$)$ for coronary heart disease, and 1.15 (0.92 to 1.43 ) and 1.63 (1.14 to 2.34) for stroke. Relative risks for cadmium and copper were 1.33 (1.09 to 1.64) and 1.81 (1.05 to 3.11) for cardiovascular disease, 1.29 (0.98 to 1.71 ) and 2.22 (1.31 to 3.74$)$ for coronary heart disease and 1.72 (1.29 to 2.28) and 1.29 (0.77 to 2.17) for stroke. Mercury had no distinctive association with cardiovascular outcomes. There was a linear dose-response relation for arsenic, lead, and cadmium with cardiovascular disease outcomes.

Conclusions: Exposure to arsenic, lead, cadmium, and copper is associated with an increased risk of cardiovascular disease and coronary heart disease. Mercury is not associated with cardiovascular risk. These findings reinforce the importance of environmental toxic metals in cardiovascular risk, beyond the roles of conventional behavioural risk factors.
Metformin exposure in first trimester of

pregnancy and risk of all or specific

congenital anomalies: exploratory case control study

Given JE, Loane $M$, Garne $M$, et al

BMJ 2018; 361:k2477, doi: 10. 1136/bmjk2477

Objective : To investigate whether exposure to metformin during the first trimester of pregnancy, for diabetes or other indications, increases the risk of all or specific congenital anomalies.

Design: Population based exploratory case control study using malformed controls. Cases of 29 specific subgroups of non genetic anomalies, and all non genetic anomalies combined, were compared with controls (all other non genetic anomalies or genetic syndromes).

Setting: 11 EUROmediCAT European congenital anomaly registries surveying 1892482 births in Europe between 2006 and 2013.

Participants: 50167 babies affected by congenital anomaly (41242 non genetic and 8925 genetic) including live births, fetal deaths from 20 weeks gestation, and terminations of pregnancy for fetal anomaly.

Main outcome measure: Odds ratios adjusted for maternal age, registry, multiple birth, and maternal diabetes status.

Results: 168 babies affected by congenital anomaly (141 nongenetic and 27 genetic) were exposed to metformin, 3.3 per 1000 births. No evidence was found for a higher proportion of exposure to metformin during the first trimester among babies with all non-genetic anomalies combined compared with genetic controls (adjusted odds ratio 0.84 , 95\% confidence interval 0.55 to 1.30). The only significant result was for pulmonary valve atresia (adjusted odds ratio 3.54, 1.05 to 12.00 , compared with non-genetic controls; 2.86, 0.79 to 10.30 , compared with genetic controls).

Conclusions: No evidence was found for an increased risk of all non-genetic congenital anomalies combined following exposure to metformin during the first trimester, and the one significant association was no more than would be expected by chance Further surveillance is needed to increase sample size and follow up the cardiac signal, but these findings are reassuring given the increasing use of metformin in pregnancy. 
Amoxicillin clavulanate versus azithromycin for respiratory exacerbations in children with bronchiectasis (BEST 2): a multicentre, double blind, non inferiority, randomized controlled trial

Goyal V, Grimwood K, Byrnes CA et al.

Lancet 2018; 392: 1197-206

Background: Although amoxicillin- clavulanate is the recommended first-line empirical oral antibiotic treatment for non-severe exacerbations in children with bronchiectasis, azithromycin is also often prescribed for its convenient once-daily dosing. No randomised controlled trials involving acute exacerbations in children with bronchiectasis have been published to our knowledge. We hypothesised that azithromycin is non-inferior to amoxicillin-clavulanate for resolving exacerbations in children with bronchiectasis.

Methods: We did this parallel group, doubledummy, double-blind, non-inferiority randomised controlled trial in three Australian and one New Zealand hospital between April, 2012, and August, 2016. We enrolled children aged 1-19 years with radiographically proven bronchiectasis unrelated to cystic fibrosis. At the start of an exacerbation, children were randomly assigned to oral suspensions of either amoxicillin-clavulanate (22.5 $\mathrm{mg} / \mathrm{kg}$, twice daily) and placebo or azithromycin ( $5 \mathrm{mg} / \mathrm{kg}$ per day) and placebo for 21 days. We used permuted block randomisation (stratified by age, site, and cause) with concealed allocation. The primary outcome was resolution of exacerbation (defined as a return to baseline) by 21 days in the per-protocol population, with a non-inferiority margin of 20\%. We assessed several secondary outcomes including duration of exacerbation, time to next exacerbation, laboratory, respiratory, and quality of life measurements, and microbiology. This trial was registered with the Australian/New Zealand Registry (ACTRN 12612000010897).

Findings: We screened 604 children and enrolled 236. 179 children had an exacerbation and were assigned to treatment: 97 to amoxicillinclavulanate, 82 to azithromycin). By day 21, $61(84 \%)$ of 73 exacerbations had resolved in the azithromycin group versus $73(84 \%)$ of 87 in the amoxiciuin-clavulanate group. The risk difference showed noninferiority $(-0.3 \%, 95 \%$ CI- 11.8 to 11.1). Exacerbations were significantly shorter in the amoxicillin-clavulanate group than in the azithromycin group (median 10 days [IQR 6-15] vs 14 days [8-16]; $p=0.014)$. Adverse events were attributed to the trial medication in $17(21 \%)$ of 82 children in the azithromycin group versus 23 (24\%) of 97 in the amoxicillin-clavulanale group (relative risk 0.9,95\% CI 0.5 to 1.5 ).

Interpretation: By 21 days of treatment, azithromycin is non-inferior to amoxicillinclavulanate for resolving exacerbations in children with non-severe bronchiectasis. In some patients, such as those with penicillin hypersensitivity or those likely to have poor adherence, azithromycin provides another option for treating exacerbations, but must be balanced with risk of treatment failure (within a 20\% margin), longer exacerbation duration, and the risk of inducing macrolide resistance. Funding Australian National Health and Medical Research Council.

\section{Invasive bacterial infections in young afebrile infants with a history of fever}

Mintegi S, Gomez B, Carro A, et al.

Arch Dis Child 2018;103:665-669.

doi:10.1136/archdischild 2017-313578

Objective: To determine the prevalence of invasive bacterial infections (IBI) pathogenic bacteria in blood or cerebrospinal fluid) in infants less than 90 days old with fever without a source related to the presence or absence of fever on arrival to the emergency department(ED).

Design: Prospective registry-based cohort study.

Setting: Paediatric ED of a tertiary teaching hospital.

Patients: We included infants less than 90 days old with a history of fever evaluated in the ED from 2003 to 2016.

Main outcomes and measures: The prevalence of IBI in patients with a history of fever who were febrile and afebrile on arrival to the ED.

Results: We included 2470 infants: 678 afebrile and 1792 febrile when evaluated in the ED. Fifty nine $(2.4 \%)$ were diagnosed with an IBI (bacteraemia 46, meningitis 7 and sepsis 6): 16 in the group of afebrile infants with a history of fever $(2.4 \%, 95 \%$ CI 1.4 to 3.8 vs 43 in the febrile group, $2.4 \%, 95 \%$ CI 1.8 to 3.2 ). Of the 16 afebrile infants with a history of fever diagnosed with an IBI, 14 were well appearing. The rate of non-IBI (pathogenic bacteria in urine or stools) was similar in both groups (15.5\% and 16.7\%).

Conclusions: The prevalence of IBI in infants: $£ 90$ days with a history of fever is similar regardless of the presence of fever on the arrival at the ED. The approach to infants with a history of fever who are afebrile in the ED should not differ from that recommended for infants who are febrile in the ED. 


\section{Fine particulate pollution and asthma exacerbations}

Bouazza N, Foissac F, Urien S, et al.

Arch Dis Child 2018;103:828 831.

doi: 10.1136/archdischild 2017-312826

Objective: As the results from epidemiological studies about the impact of outdoor air pollution on asthma in children are heterogeneous, our objective was to investigate the association between asthma exacerbation in children and exposure to air pollutants.

Methods: A database of 1264585 paediatric visits during the 2010-2015 period to the emergency rooms from 20 emergency departments (EDs) of 'Assistance Publique Hopitaux de Paris (APUP)', the largest hospital group in Europe, was used. A total of 47107 visits were classified as asthma exacerbations. Concentration of air pollutants (nitrogen dioxide, ozone, fine particulate matter (PM) with an aerodynamic diameter smaller than $10 \mathrm{um}$ (PM10) and $2.5 \mathrm{~mm}$ (PM2.5), as well as meteorological data, evolution of respiratory syncytial virus infection and pollen exposition, were collected on an hourly or daily basis for the same period using institutional databases. To assess the association between air pollution and asthma, mixed-effects quasi-Poisson regression modelling was performed.

Results: The only compound independently associated with ED visits for asthma was PM2.5 $(\mathrm{P}<10-4)$. The association between asthma exacerbation and PM2.5 was not linear, and a sigmoid function described the relationship satisfactorily. PM2.5 concentration, which gives half the maximum effect, was estimated at 13.5 $\mathrm{mg} / \mathrm{m} 3$.

Conclusions: We found an association between daily asthma exacerbation in paediatric visits to the ED and fine particulate air pollutants.

\section{Prophylactic cholecystectomy in short bowel syndrome: Is it being utilized?}

Jon S, Thompson DF, Mercer LM, et al.

The American J of Surgery 2018; $216: 7377$

Introduction: Cholelithiasis is common in patients with short bowel syndrome (SBS). Prophylactic cholecystectomy (PC) of the non-diseased gallbladder has been recommended in SBS patients when laparotomy is being undertaken for other reasons. Our aim was to determine if $\mathrm{PC}$ is being utilized.

Methods: 500 adults with SBS were seen over a 25 year period. 215 undergoing cholecystectomy prior to SBS were excluded, leaving 285 patients for evaluation.
Results: 151 (53\%) SBS patients underwent a subsequent laparotomy. 77 underwent cholecystectomy for cholelithiasis at the Ist opportunity. 27 patients underwent a PC at the Ist opportunity. 47 patients did not undergo $\mathrm{PC}$ at the Ist opportunity. $17(36 \%)$ of these 47 patients subsequently developed cholelithiasis with 7 undergoing cholecystectomy. Age, gender, diagnosis and initial BMI and need for longterm parenteral nutrition were similar in patients who had PC or did not. PC patients were more likely to have intestinal remnant length $<60 \mathrm{~cm}(59 \%$ vs $21 \%, \mathrm{p}<.05)$.

Conclusions: Overall $10 \%$ of SBS patients underwent PC. However, only $36 \%$ of eligible patients undergoing laparotomy had a PC.

\section{Differences between palpable and nonpalpable tumors in early-stage, hormone receptor positive breast cancer}

Warren SL, Bhutiani N, Agle SC, et al.

The American J of Surgery 2018; 216 : 326-30

Background: We compared characteristics and outcomes of palpable versus nonpalpable, hormone sensitive, early stage breast cancers.

Methods: Patients from the North American Fareston vs. Tamoxifen Adjuvant (NAFTA) trial were divided into palpable $(n=513)$ and nonpalpable $(\mathrm{n}=1063)$ tumor groups. Differences in pathological features, loco-regional therapy, disease free survival (DFS) and overall survival (OS) were analyzed.

Results: Patients with palpable tumors were older, had larger tumors, and higher rates of lymph node involvement. The tumors were more likely to be poorly differentiated, of high nuclear grade, and display lymphovascular invasion. After mean follow-up of 59 months, DFS and OS were significantly lower for palpable than non-palpable tumors (DFS 93.5\% vs. 98.4\%, p < 0.001, OS $88.5 \%$ vs. $95.6 \%, p<0.001)$. Controlling for age, size and nodal status, palpability was an independent factor for DFS $(\mathrm{OR}=2.56 ; 95 \% \mathrm{Cl}$, $1.37-4.79, \mathrm{p}=0.003)$ and $\mathrm{OS}(\mathrm{OR}=2.12 ; 95 \% \mathrm{CI}$ 1.38-3.28, $\mathrm{p}<0.001)$.

Conclusions: In a group of hormone sensitive, mostly postmenopausal early stage breast cancer patients, palpable tumors were more likely to have more aggressive features and metastatic potential, which translated in to a higher incidence of breast cancer-related events and worse overall survival.

\footnotetext{
A prospective cohort study of meat and fish consumption and endometriosis risk Yamamoto A, Harris HR, Allison F et al American $J$ of Obst and Gyne 2018; 219 :178 Background: Only 2 case control studies have
} 
examined the associations between consumption of meat products and endometriosis risk with inconsistent results. Consumption of animal products has the potential to influence endometriosis risk through effects on steroid hormones levels.

Objective: We sought to determine whether higher intake of red meat, poultry, fish, and seafood are associated with risk of laparoscopically confirmed endometriosis.

Study Design: A total of 81,908 participants of the prospective Nurses' Health Study II were followed up from 1991 through 2013. Diet was assessed via food frequency questionnaire every 4 years. Cox proportional hazards models were used to calculate rate ratios and $95 \%$ confidence intervals.

Results: During 1,019,294 person-years of follow up, 3800 cases of incident laparoscopically confirmed endometriosis were reported. Women consuming $>2$ servings/d of red meat had a $56 \%$ higher risk of endometriosis $(95 \%$ confidence interval, 1.22-1.99; $\mathrm{P}$ trend <.0001) compared to those consuming $<1$ serving/wk. This association was strongest for nonprocessed red meats (rate ratio, 1.57 ; 95\% confidence interval, $135-1.83$ for $>2$ servings/d vs < 1 servings /wk, Ptrend <.0001), particularly among women who had not reported infertility (Pinteraction $=$.0004). Women in the highest category of processed red meat intake also had a higher risk of endometriosis (rate ratio, $1.20 ; 95 \%$ confidence interval, $1.06-1.37$ for $>5$ servings/wk vs $<1$ serving/mo; $\mathrm{P}$ trend $=.02$ ) Intakes of poultry, fish, shellfish, and eggs were unrelated to endometriosis risk.

Conclusion: Our prospective analysis among premenopausal US nurses suggests that red meat consumption may be an modifiable risk factor for endometriosis, particularly among women with endometriosis who had not reported infertility and thus were more likely to present with pain symptoms. Well designed dietary intervention studies among women with endometriosis could help confirm this observation.

\section{Infant out come after complete uterine rupture}

Zirqi IA, Daltveit AK, Vangen S.

American J of Obst and Gyne 2018; 219 : 109

Background: Complete uterine rupture is a rare peripartum complication often associated with a catastrophic outcome for both mother and child. However, little has been written based on large data sets about maternal and infant outcome after complete rupture. This is partly due to the rarity of the event and the serious maternal and infant outcome; it is also partly due to the use of international diagnostic codes that do not differentiate between the less catastrophic partial rupture and more catastrophic complete uterine rupture. As uterine rupture is expected to increase due to increased cesarean delivery rates worldwide, it is important to know more completely about the outcome following complete uterine rupture.

Objective: We sought to explore risk factors associated with poor infant outcome in cases of complete uterine rupture.

Study Design: This population based study used data from the Medical Birth Registry of Norway, the Patient Administration System, and medical records. We included births with complete uterine rupture after start of labor in all maternity units in Norway during the period of 1967 through 2008 ( $\mathrm{n}=244$ births), identified among 2,455,797 births. Uterine ruptures were identified and further studied through a review of medical records. We estimated the associations between infant outcomes and demographic and labor risk factors using logistic regression analyses. Odds ratios with $95 \%$ confidence intervals for each risk factor were determined after adjustment for demographic factors and period of birth. The main outcome measure was infant outcome: healthy infant, intrapartum/infant deaths, hypoxic ischemic encephalopathy, and admission to the neonatal intensive care unit.

Results: We identified 109(44.7\%) healthy infants, $56(23.0 \%)$ infants needing neonatal intensive care unit admission, 64(26.2\%) intrapartum/infant deaths, and $15(6.1 \%)$ infants with hypoxic ischemic encephalopathy. The highest number of intrapartum/infant deaths occurred in 1967 through 1977 (51.65\%) and the fewest in 2000 through 2008 (15.0\%). Unscarred uterine ruptures did not significantly increase intrapartum/infant deaths compared to scarred uterine ruptures. Placental separation and/or fetal extrusion had the highest odds ratio for intrapartum/infant deaths (odds ratio, 17.9; 95\% confidence interval, 7.5-42.4). Time to delivery interval $<20$ minutes resulted in fewest intrapartum/infant deaths (9.9\%), although there were 2 deaths at 10 minute interval. Time to delivery $>30$ minutes vs $<20$ minutes increased risk of death (odds ratio, 16.7; 95\% confidence interval, 6.4 43.5).

Conclusion: Intrapartum/infant death after complete uterine rupture decreased significantly over the decades. Time to delivery $>30$ minutes and placental separation and/or fetal extrusion had the highest association with intrapartum/infant deaths after complete uterine rupture. Time to delivery $<20$ minutes limited the incidence of intrapartum/infant deaths. 